\title{
Research Status and Development Trend of Optimal Sensor Placement
}

\author{
Zhaolan Wei ${ }^{1,}$ a , Jing $\mathrm{Xia}^{2, \mathrm{~b}, *}$ \\ ${ }^{1}$ College of Civil Engineering, Sichuan Agricultural University, Dujiangyan, Sichuan,611830, \\ China \\ ${ }^{2}$ College of Civil Engineering, Sichuan Agricultural University, Dujiangyan, Sichuan,611830, \\ China \\ aweifei0722@163.com, ${ }^{b}$ xia2273789637@163.com
}

Keywords: optimal sensor placement, traditional optimization algorithms, random optimization algorithm, development trend

\begin{abstract}
Sensor optimization is a combinatorial optimization problem. It has important significance in structural health monitoring and damage identification. The choice of sensor optimization method is directly related to the efficiency and feasibility of optimization calculation. At present, the optimization algorithm is mainly divided into two categories, the traditional optimization algorithm and the stochastic algorithm. The traditional optimization algorithms is a deterministic search model based on numerical calculation, and random optimization algorithm is a probabilistic random search model based on non-numerical calculation. In this paper, common algorithms are introduced, and their existing problems are analyzed. Finally, some shortages and development trends in the research of optimal sensor placement are pointed out.
\end{abstract}

\section{Introduction}

The sensor optimization arrangement has attracted much attention and has an important position in many fields, especially in the field of modal parameter identification. The modal parameter identification (natural frequency, damping ratio and mode shape) is performed by the sensor's measured structural response. Although with the development of technology, the cost of sensors is rapidly declining, the number of affordable sensors is still a problem in long-span bridges and high-rise buildings. When using a limited number of sensors, there is inevitably uncertainty in the identification parameters. Therefore, the location of the sensor and the number of sensors used is a key task. Research has shown that optimal sensor placement is critical to improve the accuracy of modal parameter identification. In the aspect of system function, in order to describe the continuous function, the limited sensor is usually used to obtain the discrete information and the discrete information is used to describe the continuous function. Therefore, a good sensor arrangement scheme is particularly important. Therefore, the placement of the sensors should meet the following 
two goals: 1) information that best reflects the spatial structure; and 2) be sensitive enough to changes in the state of the spatial structure.

\section{Research Status of Optimal Sensor Placement}

Sensor optimization is a combinatorial optimization problem, and its solution is still one of the research hotspots. The choice of optimization method is directly related to the efficiency and feasibility of optimization calculation. At present, a large number of domestic and foreign scholars have studied it, and have obtained a variety of optimized layout methods, which are mainly divided into two categories: the traditional optimization algorithm based on the deterministic search model of numerical calculation and the random optimization algorithm based on a probabilistic random search model based on non-numerical calculation. The goal is to use a limited number of sensors to obtain the maximum amount of information.

\subsection{Traditional Optimization Algorithms}

\subsubsection{Effective Independence Method}

Kammer [1] proposed an effective independence (EI) method for modal identification, which tends to maximize the determinant of the Fisher information matrix (FIM). According to the ranking of the contribution of each measurement point to the independence of the target mode matrix, the candidate measurement points with the smallest contribution to the rank are deleted in order to optimize the Fisher information matrix so that the modal vectors of interest are kept as linear as possible. This method also has its limitations. It has a large amount of calculation, it easily leads to the aggregation of measurement points, and the signal-to-noise ratio of the measurement point is low, causing the loss of important modal information. So, to improve on this method, many optimization algorithms are extended. Using QR decomposition, modal kinetic energy method, cluster and other methods, starting from all measuring points, selecting candidate measuring points, and then using effective independent method to filter the initial measuring points to reduce the workload. Udwadia [2] proposed the Fisher information standard in parameter identification, where the optimal configuration corresponds to maximizing FIM traces, maximizing the amount of information. He et al. [3] proposed a distance coefficient-effective independent method based on the distance coefficient correction information matrix, which improved the measurement point aggregation and other issues. Based on the distance coefficient, Dong et al. [4] proposed a method to modify the model error covariance in the Fisher information matrix using a modal contribution and distance coefficient, so as to achieve a reasonable modification of the Fisher information matrix. Cheng et al.[5] used the frequency response function of the measurement point as the driving-point residual weighted effective independent distribution vector, and proposed an improved effective independent method for the optimal placement of sensors, taking into account the measurement point energy size, and improving the signal-to-noise ratio at the measurement point. The traditional optimization algorithms have certain practicality, and the resulting layout scheme also has a rationality. However, these methods all have their own limitations, and the obtained results are often sub-optimal solutions, which cannot guarantee the minimum error between the theoretical and measured information.

\subsubsection{Sequence Method}

The basic idea of the sequence method is to minimize the non-diagonal elements of the MAC matrix and make the information between the measurement points independent. The sequence 
method can be divided into gradual accumulation method and gradual elimination method. The gradual elimination method gradually removes those points that have the smallest contribution to the objective function from the remaining measurement point locations until the desired number of measurement points remains. The stepwise accumulation method happens to be the opposite of this, and the QR decomposition is used to select the initial measurement point from Start from the initial measurement point and gradually increase the measurement point until the desired number is reached. This method is simple and fast, but regardless of which method, the final result is a suboptimal solution.

\subsubsection{Modal Kinetic Energy Method and Model Reduction Method}

The modal kinetic energy method proposed by Heo [6] is a relatively quantized method. It changes the traditional engineer's dependence on the selection of the location sensor with larger vibration mode. It combines the mass matrix and the modal vector, and selects the location sensor with larger modal kinetic energy. The maximum response of each measuring point is roughly calculated, and the signal-to-noise ratio (SNR) is increased, it is suitable for the environment with large noise and is generally used for the selection of initial measurement points for large structures. Debnath et al. [7] proposed the modal contribution of the output energy and applied it to the evaluation of the sensor layout, breaking through the traditional kinetic energy method. The Guyan model reduces the [8] which contains the constraint equations with the primary and secondary coordinates into the kinetic energy or strain energy of the system, produces the reduced mass or stiffness matrix, iterates by successive iteration, and preserves the main coordinates of the modal reactions as the measurement point arrangement. This method also has some limitations. After iterating, only low-order modes exist, and the higher-order modes are reduced, which is greatly affected by the meshing.

\subsection{Random Optimization Algorithm}

In recent decades, with the development of bionics, people gradually begin to pay attention to the phenomena of nature and get inspiration from it. Random class algorithm is a method to simulate biological and physical processes based on this. The random class algorithm in the sensor optimization arrangement mainly includes genetic algorithm, neural network method, monkey group algorithm and simulated annealing method. It can well solve the restriction of constraint conditions in the combinatorial optimization problem, and cannot fall into the local optimal. Genetic algorithm simulates the biological evolution process and optimizes it through selection, crossover and mutation operations. It has better robustness and versatility, faster convergence speed and more reliable stability, but there are also premature issues. In order to overcome the premature problem, Gao et al. [9] introduced the simulated annealing algorithm with strong local search ability into the genetic algorithm. Yuan et al. [10] combined the genetic algorithm and the neural network algorithm to optimize the acceleration sensor, established a neural network model with double hidden layers for training. The trained model was used to search the optimal value based on genetic algorithm. Zhang et al. [11] introduced the nested partitioning algorithm into the genetic algorithm, divided the fundamental solution into different regions, and finally used the genetic algorithm to search in each region to obtain the optimal solution. The monkey group algorithm designed three search processes for solving large-scale and multi peak optimization problems by simulating several actions of climbing, looking and jumping during the mountain climbing process in the natural world. It is suitable for the optimization of large span bridge sensors. The monkey group algorithm designed three search processes for solving large-scale and multi-peak value problems by simulating several actions of climbing, looking and jumping during the monkey group climbing mountain process in the 
natural world. It is suitable for the optimization of large span bridge sensors. Li et al. [12] applied the monkey algorithm to the long-span continuous rigid frame bridge, showing its applicability. Because this kind of algorithm adopts the probabilistic random search model of non numerical calculation, it breaks through the traditional optimization method based on the deterministic search mode with numerical calculation. So it is not easy to converge to the local optimal solution, and it has a broad development prospect in the optimization of sensor layout in large spatial structure analysis. As a new tool, although this type of algorithm has better parallelism and global search, its search ability, speed of convergence and stability still remain to be further studied. The efficiency and reliability of this algorithm still need to be improved, and its programming and operation are worth further good.

\subsection{Other Methods}

Information theory is an applied mathematics subject which studies information, information entropy, communication system and data transmission by probability theory and mathematical statistics. It has been widely applied in the field of parameter identification and modal identification. Information entropy proposed by Papadimitriou [13] is a method of sensor optimization based on information theory. It integrates Bayesian theory into information entropy as a direct measure of model parameter uncertainty. Minimize information entropy as an objective function to optimize the sensor placement scheme, where a certain number of sensors obtain the maximum amount of information. Zhang et al. [14] people combine information entropy with genetic algorithm and use information entropy as the fitness function of genetic algorithm. Minimize the information entropy index in all possible sensor configuration combinations, and get the optimal layout of the sensor. Information entropy is often used in combination with genetic algorithms. When there are more combinations, exhaustive search cannot be used. Artificial intelligence-based genetic algorithm has stronger global optimization ability than traditional gradient-based optimization algorithms.

In addition to the optimization algorithm, the amount of information acquired by the sensor also depends on the load conditions, and the output response of the structure changes significantly under different input loads. Therefore, the optimal sensor configuration usually depends on the load conditions. Li et al. [15] proposed a sensor placement method considering load and structural response and showed improved identification performance. Brehm [16] determines the best position of the reference sensor under white noise excitation and multi-pulse excitation. Although many innovative theories have been proposed in the area of sensor optimization, there is still considerable uncertainty in the determination of the number and location of sensors for obtaining sufficient information.

\section{Disadvantages}

In the field of bridge health monitoring and damage identification, how to make the sensor acquire abundant modal information needs in-depth study. Although there are many sensor optimization algorithms, there are still many problems, mainly as follows:

(1) The selection of the number of sensors must take into consideration both economic and optimization factors. The existing optimization arrangements are based on the determination of the number of sensors. Therefore, it is still a difficult point to determine the appropriate number of sensors, which needs further research.

(2) the optimal sensor placement is mainly focused on the optimization of the dynamic test. There are few studies on the static test, the static and dynamic test and the influence of the environment on the measurement point. However, in the health monitoring of the actual engineering structure, the factors such as displacement, deflection and strain of static test are often considered. In fact, the two 
systems have not yet formed a more systematic theory, and there is no corresponding standard and standard guidance.

(3) There is no general and effective evaluation criteria for the evaluation of optimal sensor placement, and there is no corresponding standard. The evaluation criteria are only partially unified in theory. Therefore, it is necessary to establish a comprehensive sensor optimization evaluation system and formulate corresponding specifications.

\section{Solutions}

Sensor optimization is a combinatorial optimization problem, the integer Planning Problem. Due to the difficulty of combinatorial optimization, its solution is still one of the hot topics in the research. Therefore, it is necessary to explore an optimization method with better parallelism, global search, fast convergence speed and fewer iterations, so as to improve the computational efficiency and improve the reliability.

(1) At present, the sensor optimization algorithm considers the factors too single, the optimization result is skewed popularity, and the optimal measurement point selection cannot be achieved. Therefore, different methods are merged to form a new hybrid algorithm. Various influencing factors are jointly considered to obtain the optimal solution. For example, A Hybrid Algorithm Based on EI and MAC, A Hybrid Algorithm Based on genetic algorithms and MAC .

(2) The sensor optimization arrangement is eventually applied to the project. Therefore, the optimization theory is converted into software, which is not only the most direct test of practice but also convenient and quick to use. Many scholars have begun to pay attention to how to improve the computational efficiency of optimization algorithms in sensor optimization arrangements, reduce the amount of iterative calculations, and perform sensor optimization toolkit development.

(3) For large space structures, there are not only many structural uncertainties, but also work in a complex natural environment. These unfavorable conditions bring certain difficulties to the monitoring of the structure. Therefore, in the sensor layout It is necessary to determine the best deployment plan based on a combination of multiple methods, taking into account specific structural features and measurement conditions.

\section{Conclusion}

Optimal placement of sensors is a key step in the rationality, accuracy, long-term and economic performance of bridge structural health monitoring system. Improper sensor arrangement will affect the accuracy of the identification parameters, and the sensor itself needs a certain cost, and the cost of the data acquisition and processing equipment is also high. From the economic point of view, we want to use as few sensors as possible to provide as much information as possible. Therefore, determining the optimal number of sensors and configuring them in optimal locations is of great practical value.

\section{References}

[1] Kammer D C. Sensor Placement for On-Orbit Modal Identification and Correlation of Large Space Structures[J]. Journal of Guidance, Control and Dynamics, 1991, 14(9):251 -259.

[2] Udwadia F E. Methodology for Optimum Sensor Locations for Parameter Identification in Dynamic Systems[J]. Journal of Engineering Mechanics, 1994, 120(2):368-390.

[3] He L J, Lian J J, Ma B, et al. Optimal sensors placement for large space structures based on distance coefficient-effective independence method [J]. Journal of Vibration and Shock, 2013, 32(16):13-18.

[4] Dong K L, Ma J J, Yin H, et al. Modify covariance of Fisher information matrix in sensor placement [J]. Information and Control, 2018, 47(1):68-74. 
[5] Chen J Q, Yan W M, Chen Y J, et al. An improved effective method for optimal sensor placement[J]. Vibration, test and diagnosis, 2012, 32(5):812-816.

[6] Heo G, Wang M L, Satpathi D. Optimal transducer placement for health monitoring of long span bridge[J]. Soil Dynamics \& Earthquake Engineering, 1997, 16(7-8):495-502.

[7] Debnath N, Dutta A, Deb S K. Placement of sensors in operational modal analysis for truss bridges[J]. Mechanical Systems \& Signal Processing, 2012, 31(8):196-216.

[8] R. J. GUYAN. Reduction of stiffness and mass matrices[J]. AIAA Journal, 1965, 3(2):380-380.

[9] Gao W C, Xu M J, Liu W. Optimal placement of sensors based on genetic algorithm[J]. Journal of Harbin Institute of Technology. 2008, 40(1):9-11.

[10] Yuan C, Tang C T, Li W Z. An Optimal placement method of bridge acceleration sensor based on neural network and genetic algorithm [J]. Journal of Xihua University (Natural Science Edition), 2018(2).

[11] Zhang P Y, Zhang X D, Chen W D, et al. Optimal placement of sensors for long-span bridges based on nested-stacking genetic algorithm[J]. Journal of Wuhan University of Technology (Transportation Science \& Engineering), 2016, 40(4):745-749.

[12] Li X, Ni F T, Sun W G, et al. Research on optimal sensor placement of continuous rigid frame bridge based on monkey group algorithm[J]. Highway, 2016(3):65-69.

[13] Papadimitriou C, Beck J L, Au S K. Entropy-Based Optimal Sensor Location for Structural Model Updating[J]. Journal of Vibration \& Control, 2000, 6(5):781-800.

[14] Zhang J P, Yin T, Zhu H P, et al. Optimal sensor configuration based on spectral finite element method and information entropy[J]. Journal of Vibration and Shock, 2016, 35(2):76-81.

[15] Li D S, Li H N, Fritzen C P. Load dependent sensor placement method: Theory and experimental validation[J]. Mechanical Systems \& Signal Processing, 2012, 31(8):217-227.

[16] Brehm M, Zabel V, Bucher C. Optimal reference sensor positions using output-only vibration test data[J]. Mechanical Systems \& Signal Processing, 2013, 41(1-2):196-225. 